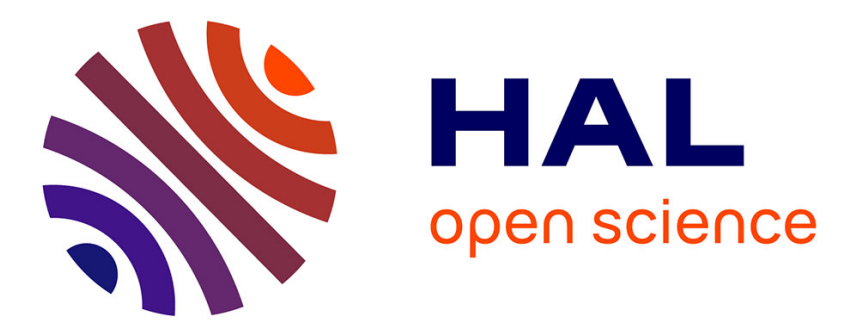

\title{
Nonlinear pulse shaping by coherent addition of multiple redshifted solitons
}

\author{
F.R. Andresen, J.M. Dudley, D. Oron, C. Finot, H. Rigneault
}

\section{To cite this version:}

F.R. Andresen, J.M. Dudley, D. Oron, C. Finot, H. Rigneault. Nonlinear pulse shaping by coherent addition of multiple redshifted solitons. Journal of the Optical Society of America B, 2011, 28 (7), pp.1716-1723. 10.1364/JOSAB.28.001716 . hal-00655054

\section{HAL Id: hal-00655054 https://hal.science/hal-00655054}

Submitted on 5 May 2021

HAL is a multi-disciplinary open access archive for the deposit and dissemination of scientific research documents, whether they are published or not. The documents may come from teaching and research institutions in France or abroad, or from public or private research centers.
L'archive ouverte pluridisciplinaire HAL, est destinée au dépôt et à la diffusion de documents scientifiques de niveau recherche, publiés ou non, émanant des établissements d'enseignement et de recherche français ou étrangers, des laboratoires publics ou privés. 


\title{
Nonlinear pulse shaping by coherent addition of multiple redshifted solitons
}

\author{
Esben Ravn Andresen, ${ }^{1}$ John M. Dudley, ${ }^{2}$ Dan Oron, ${ }^{3}$ Christophe Finot, ${ }^{4}$ and Hervé Rigneault ${ }^{1, *}$ \\ ${ }^{1}$ Institut Fresnel, Aix-Marseille Université, CNRS, Domaine Universitaire de St. Jérôme, 13397 Marseille Cedex 20, France \\ ${ }^{2}$ Laboratoire d'Optique P. M. Duffieux, Institut France Comté Electronique Mécanique Thermique et Optique-Sciences \\ et Technologies CNRS, 16 Route de Gray, 25030 Besançon Cedex, France \\ ${ }^{3}$ Department of Physics of Complex Systems, Weizmann Institute of Science, Rehovot 76100, Israel \\ ${ }^{4}$ Laboratoire Interdisciplinaire Carnot de Bourgogne, CNRS, Université de Bourgogne, 21078 Dijon, France \\ ${ }^{*}$ Corresponding author: herve.rigneault@fresnel.fr
}

\begin{abstract}
The injection of a phase- and amplitude-shaped pulse into a photonic-crystal fiber provides additional degrees of freedom that can significantly influence the nature of nonlinear propagation and nonlinear and dispersive interactions. This strong sensitivity of nonlinear effects-particularly the Raman soliton self-frequency shiftgreatly extends the parameter space available to generate tailored output fields for applications such as microscopic imaging. By numerical simulations, we identify the relevant interpulse interactions, and we experimentally demonstrate the additional capabilities of this nonlinear pulse-shaping method.
\end{abstract}

\section{INTRODUCTION}

Nonlinear propagation and spectral broadening in photoniccrystal fiber (PCF) has now been extensively studied and applied for diverse applications including frequency metrology, imaging, and spectroscopy [1,2]. For some applications, the generation of broadband spectra is not itself a requirement, and what is more important is the generation of high-brightness spectral components covering particular wavelength ranges. In this case, the absolute bandwidth span of the generated supercontinuum is less important than the characteristics of the spectral components of interest. This parameter regime can be referred to as a class of "nonlinear pulse shaping" where the preshaping of an input pulse in phase and amplitude can be used to exert control over the PCF spectral output.

The general setup for nonlinear pulse shaping is sketched in Fig. 1, where a comparison with conventional phase and amplitude shaping $[\underline{3}, \underline{4}]$ is also given. This kind of setup, utilizing a phase and amplitude shaper before an optical fiber, was used in [므묘. Of course, numerous examples of nonlinear pulse shaping have been demonstrated, albeit under various other names; common to all though is that the phase or amplitude of the PCF input is controlled in some way, though not necessarily by a phase and amplitude shaper. Examples using only a single input pulse are optical power control of the soliton self-frequency shift (SSFS) [9-11] and spectral compression by self-phase modulation of a negatively chirped pulse [12-14], which have found application in nonlinear micro-

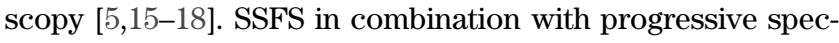
tral narrowing upon redshift in a dispersion-tailored PCF was also demonstrated [19]. Finally, fiber-generated supercontinua have been controlled to some degree by the shape of the input pulse [묵, $\underline{20}-\underline{22}]$.
Recently, the prospect of temporal compression based on the addition of several femtosecond redshifted solitons produced in a PCF was raised [23]. Two different solitons with different frequencies were coherently added to yield a broader spectrum allowing temporal compression to a shorter duration relative to the constituent solitons. Reference [5] focused purely on spectral shaping by addition of redshifted solitons; several solitons with different frequencies were added to form a broad PCF output spectrum. These papers underline the clear and current interest in developing a more complete picture of how control of the nonlinear propagation can be used to yield tailored output fields with characteristics specifically suited to particular applications.

In this paper, our purpose is to explore in detail the nonlinear shaping processes relevant to experiments such as those described in [5,23]. Here it is soliton formation and the subsequent SSFS that are the primary nonlinear effects taking place in the PCF. A particular aim is to expand the previous, mainly theoretical, work on interactions between solitons [24-39] to a predominately experimental and applied regime. This topic has, as far as we can see, not yet been fully treated in the literature on fiber solitons, which tends to focus more on soliton collisions [33,40-49] or the so-called bound pairs, bound states of solitons, or soliton molecules $[28,34,35,37-39]$. In our case, we aim to focus specifically on realistic conditions where the initial pulses are not perfect solitons, in which case two new interactions must be considered, namely the interaction between a decelerating, leading soliton and the dispersive residues shed by trailing solitons and the interaction between the pulses before they have shed a fundamental soliton. By numerical simulations and experiments, we will consider in detail the possible interactions involved in realistic experiments. Our results demonstrate the additional frequency-conversion capabilities of nonlinear 
Phase and amplitude shaping:
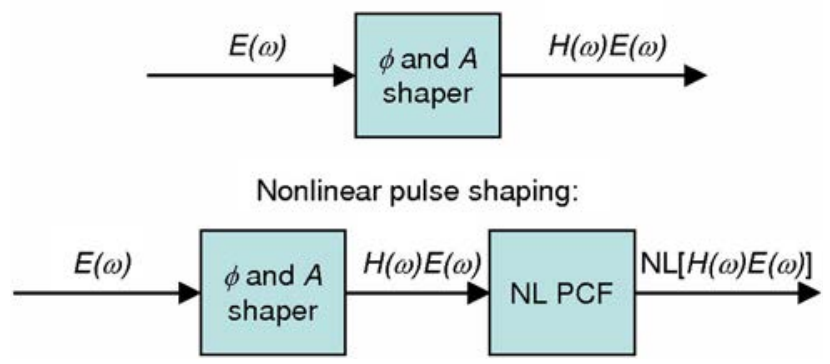

Fig. 1. (Color online) Schemes (top) for phase and amplitude shaping and (bottom) for nonlinear pulse shaping.

pulse shaping compared to traditional phase and amplitude shaping.

\section{MATERIALS AND METHODS}

\section{A. Numerical Code}

The numerical simulations used a standard generalized nonlinear Schrödinger equation model $[\underline{1}, 11]$. Our aim was to realistically describe the experiments performed using the setup in Fig. 2; thus, we used the realistic Raman response and the full dispersion curve of the fiber as detailed in Table 1 . The PCF has a single zero-dispersion wavelength at $745 \mathrm{~nm}$.

We consider initial conditions of a pair of identical pulses $E_{0}$ separated in time by $\Delta t$ and with relative phase $\Delta \phi$, i.e.,

$$
E_{\text {pair }}(t)=E_{0}(t)+E_{0}(t-\Delta t) e^{i \Delta \phi} .
$$

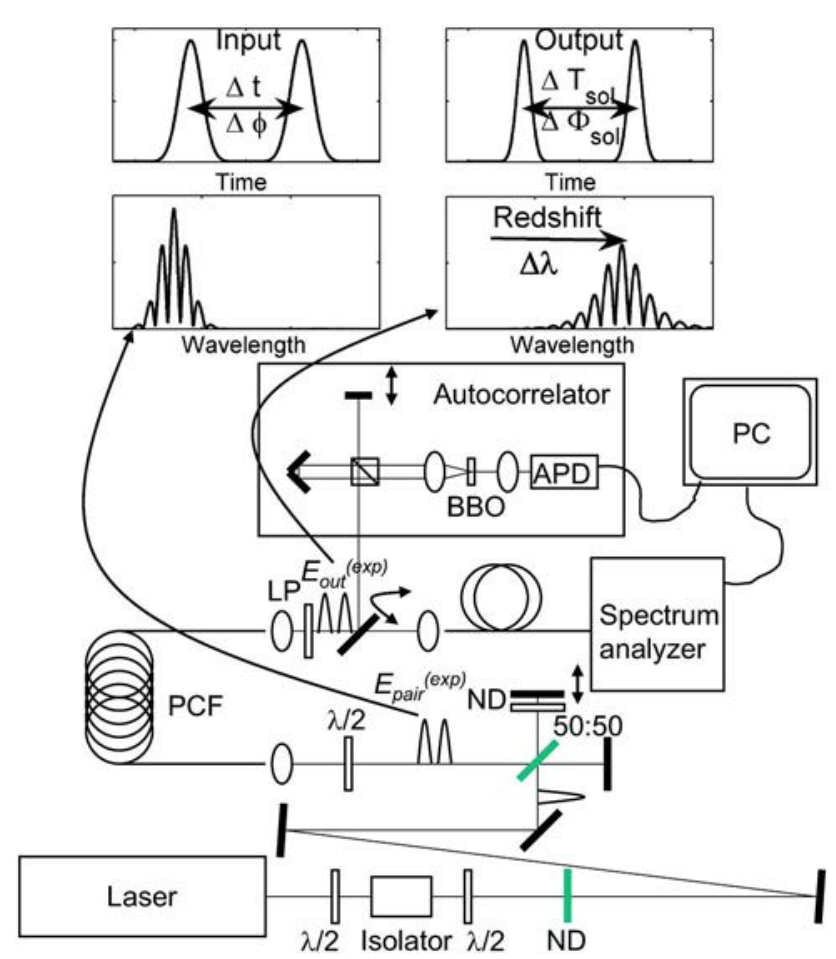

Fig. 2. (Color online) Sketch of the experimental setup. Inset graphs show sketches of (left) the input pulse pair temporal envelope and spectrum and (right) similar sketches for the output soliton pair. $\mathrm{BBO}, \beta$-barium borate; APD, avalanche photodiode; PC, personal computer; LP, long-pass filter; ND, neutral density filter.
Table 1. PCF Parameters Used at $\lambda=1035 \mathrm{~nm}$

\begin{tabular}{cc}
\hline$\beta_{2}$ & $-8.18299 \cdot 10^{4} \mathrm{fs}^{2} / \mathrm{m}$ \\
$\beta_{3}$ & $1.61038 \cdot 10^{5} \mathrm{fs}^{3} / \mathrm{m}$ \\
$\beta_{4}$ & $-1.95205 \cdot 10^{5} \mathrm{fs}^{4} / \mathrm{m}$ \\
$\beta_{5}$ & $2.07369 \cdot 10^{5} \mathrm{fs}^{5} / \mathrm{m}$ \\
$\beta_{6}$ & $6.05655 \cdot 10^{4} \mathrm{fs}^{6} / \mathrm{m}$ \\
$\beta_{7}$ & $1.34857 \cdot 10^{6} \mathrm{fs}^{7} / \mathrm{m}$ \\
$\beta_{8}$ & $-7.54952 \cdot 10^{6} \mathrm{fs}^{8} / \mathrm{m}$ \\
$\beta_{9}$ & $1.70524 \cdot 10^{7} \mathrm{fs}^{9} / \mathrm{m}$ \\
$\beta_{10}$ & $-4.71402 \cdot 10^{6} \mathrm{fs}^{10} / \mathrm{m}$ \\
$\gamma$ & $0.079(\mathrm{~W} \cdot \mathrm{m})^{-1}$ \\
\hline
\end{tabular}

To model nonideal soliton input fields, we consider different shapes of $E_{0}$ and parameters as listed in Table 2; the table also lists the corresponding section below where we present the results. The soliton number $N=\sqrt{\left(\tau^{2} \gamma P_{0}\right) /\left(1.763^{2}\left|\beta_{2}\right|\right)}$ is chosen such that each $E_{0}$ on its own approximately produces the same output of a single soliton redshifted by $\Delta \lambda=45 \mathrm{~nm}$ to $1080 \mathrm{~nm}$ at the output of the PCF. The time frame was chosen to be stationary at $\lambda=1035 \mathrm{~nm}$ and centered on $E_{0}(t)$.

From the PCF output field $E_{\text {out }}$ resulting from the numerical propagation of $E_{\text {pair }}$, the solitonic part can be identified and the relative delay $\Delta T_{\text {sol }}$, defined as the peak-peak separation, of the created soliton pair be found for different values of $\Delta \phi$, which we vary over the range 0 to $2 \pi$. The resulting $\Delta T_{\text {sol }}$ versus $\Delta \phi$ will form the basis for the comparison of the simulations with the experimental measurements.

\section{B. Experimental Setup}

A sketch of the experimental setup is shown in Fig. 2. The laser used to do the experiments is a t-pulse SESAM-modelocked, diode-pumped ytterbium laser from Amplitude Systems $(50 \mathrm{MHz}, 1035 \mathrm{~nm}, 150 \mathrm{fs})$. The laser pulses are sent through a Michelson interferometer, acting as the phaseand amplitude-shaping device, to create from the laser pulse $E_{\text {pair }}^{(\text {exp })}$. A mirror in one arm is mounted on a delay stage with a piezoelectric transducer, allowing us to set the initial separation $\Delta t$ as well as the initial relative phase $\Delta \phi$ of the pulse pair. The resulting pulse pair is then

$$
E_{\mathrm{pair}}^{(\exp )}(t)=E_{0}(t)+E_{0}\left(t-\Delta t+\frac{\Delta \phi \lambda}{2 \pi c}\right) e^{i \Delta \phi} .
$$

A neutral-density filter in one arm allows to balance the energy of the constituent pulses. $E_{\text {pair }}^{(\exp )}$ is sent through a $72.5 \mathrm{~cm}$ long PCF (NL-2.0-745-02, Blaze Photonics) in which the majority of the energy of each pulse goes to form a fundamental soliton with pulse energy of around $50 \mathrm{pJ}$ and duration FWHM $\tau_{\text {sol }}=60 \mathrm{fs}$, which redshifts upon propagation due to the SSFS. The PCF output $E_{\text {out }}^{(\exp )}$ is characterized by a noncollinear, background-free autocorrelator and a spectrum analyzer (Ando AQ-6315A), allowing us to measure the

Table 2. Initial Conditions Used in the Simulations

\begin{tabular}{ccccc}
\hline Subsection & $E_{0}(t)$ & $\tau(\mathrm{fs})$ & $P_{0}(\mathrm{~W})$ & $N$ \\
\hline $3 . \mathrm{A}$ & $\sqrt{P_{0}} \operatorname{sech}(-1.763 t / \tau)$ & 44 & 1710 & 1.0 \\
$\frac{3 . \mathrm{B}}{3 . \mathrm{C}}$ & $\sqrt{P_{0}} \exp \left(-2 \ln 2 t^{2} / \tau^{2}\right)$ & 140 & 600 & - \\
\hline & $\sqrt{P_{0}} \operatorname{sech}(-1.763 t / \tau)$ & 140 & 600 & 1.9 \\
\hline
\end{tabular}


dependent parameter $\Delta T_{\text {sol }}$. The independent parameters $\Delta t$ and $\Delta \phi$ can be found by bypassing the PCF and sending the PCF input directly into the spectrum analyzer.

\section{RESULTS: SIMULATIONS}

\section{A. Soliton Pair}

We now consider the case where a soliton pair is propagated through the PCF. Initially, we set the Raman response of the PCF to zero and ran the simulation. The obtained results closely reproduced the analytical model of the soliton force (strictly speaking, the Kerr-mediated interaction between interfering solitons) in [24,25]. The simulation predicted attraction for $\Delta \phi=0$ and repulsion for $\Delta \phi=\pi$. In this case, the relative phase of the soliton pair does not change along the length of the PCF, so that the integrated interaction observed after the PCF $\left(\Delta T_{\text {sol }}-\Delta t\right)$ is directly proportional to the soliton force. So the attractive soliton force for $\Delta \phi=0$ leads to a $\Delta T_{\text {sol-decreasing interaction and vice versa. The recorded }}$ $\Delta T_{\text {sol }}$ versus $\Delta \phi$ curve is displayed in Fig. $\underline{3(\mathrm{e})}$ and will be used for comparison later.

We proceed to the case of realistic Raman response of the PCF. If there were no interaction between the constituent solitons of $E_{\text {pair }}, E_{\text {out }}$ would still comprise identical solitons redshifted relative to the input, and there would be a correction to the relative phase due to the phase accumulated by the solitons while undergoing the SSFS, $\Delta \Phi_{\text {sol }}=\Delta \phi+\Delta \Phi_{\mathrm{SSFS}}$, $\Delta \Phi_{\mathrm{SSFS}}=2 \pi c \Delta \lambda \Delta t / \lambda^{2}$.
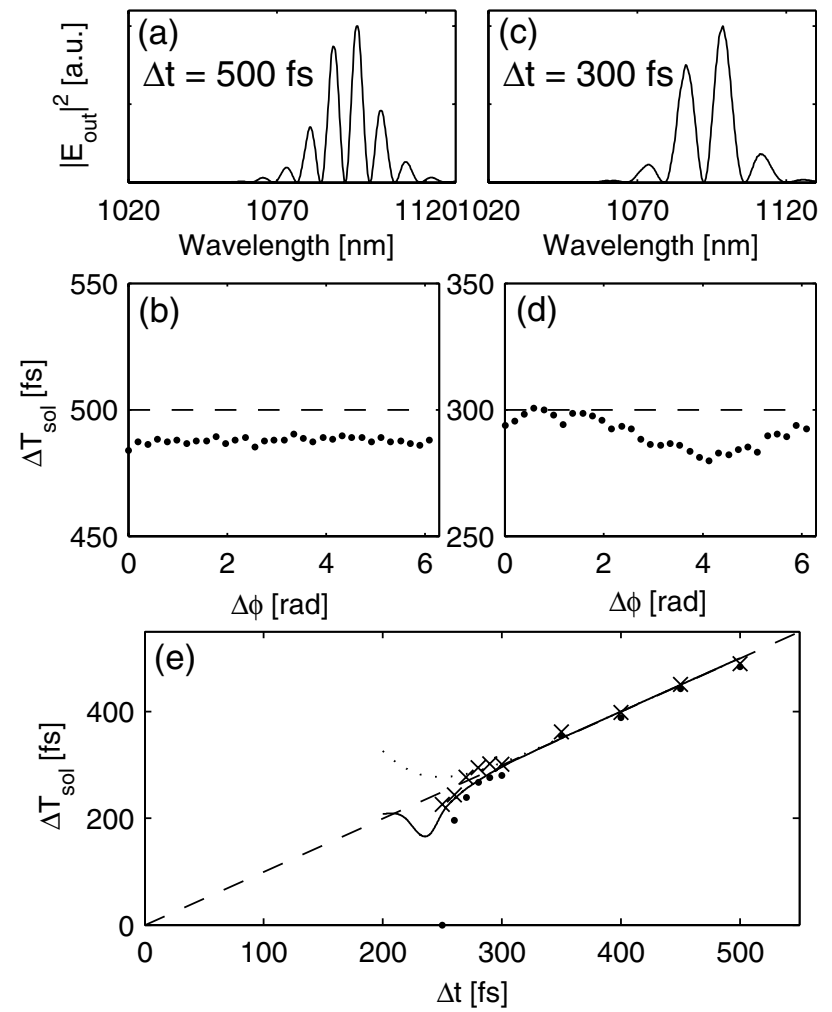

Fig. 3. Simulation; soliton pair with $\tau=44 \mathrm{fs}, \lambda=1035 \mathrm{~nm}$, and $P_{0}=1710 \mathrm{~W}$. Sample output spectra and output relative delay $\Delta T_{\text {sol }}$ versus initial relative phase $\Delta \phi$ for initial relative delay (a), (b) $\Delta t=500 \mathrm{fs}$, (c), (d) $\Delta t=300 \mathrm{fs}$, (e) $\min \left[\Delta T_{\mathrm{sol}}\right]$ (dots) and $\max \left[\Delta T_{\text {sol }}\right]$ (crosses) versus $\Delta t, \min \left[\Delta T_{\text {sol }}\right]$ (solid) and $\max \left[\Delta T_{\text {sol }}\right]$ (dotted) for Raman response set to zero, and the noninteracting case (dashed).
However, the redshifting solitons do interact, but there still is a region where the solitons can be assumed to be noninteracting. This is the case in Figs. 3(a) and 3(b), where $\Delta t=500 \mathrm{fs}$. As can be seen in Fig. $3\left(\overline{\mathrm{a})}, \Delta T_{\text {sol }}\right.$ is unchanged when $\Delta \phi$ is changed, and $\Delta \Phi_{\text {sol }}$ (not shown) is a linear function of $\Delta \phi$, completely analogous to the case of noninteracting solitons.

The situation changes when $\Delta t$ decreases. In Figs. 3(c) and

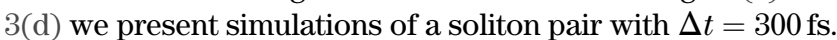
As can be seen from Fig. $3(\mathrm{~d}), \Delta T_{\text {sol }}$ now varies with $\Delta \phi$, $\min \left[\Delta T_{\text {sol }}\right]$ is at $\Delta \phi=1.3 \pi, \overline{\text { and }} \max \left[\Delta T_{\text {sol }}\right]$ is at $\Delta \phi=0.2 \pi$. For these parameters, $\Delta \Phi_{\mathrm{SSFS}} \approx 3.6 \cdot(2 \pi)$, so the soliton force changes sign repeatedly along the $\mathrm{PCF}$, which means that $\left(\Delta T_{\text {sol }}-\Delta t\right)$ is no longer proportional to the soliton force; the local, instantaneous attraction or repulsion cannot be taken as representative for the integrated interaction. This was pointed out in [36].

We perform a series of simulations like the one above for a number of $\Delta t$ and $\Delta \phi$ in the range 0 to $2 \pi$. The results are summarized in Fig. 3(e). The points plotted are $\min \left[\Delta T_{\text {sol }}\right]$ and $\max \left[\Delta T_{\text {sol }}\right]$ (when mentioning these, we assume fixed $\Delta t$ ). The differences between the $\Delta T_{\text {sol }}$ in the presence and absence of SSFS are introduced by the SSFS. In presence of SSFS, the interaction becomes $\Delta T_{\text {sol-decreasing at }}$ $\Delta t<270$ fs. In addition, $\left(\max \left[\Delta T_{\text {sol }}\right]-\min \left[\Delta T_{\text {sol }}\right]\right)$ is smaller than was the case when the Raman response was set to zero. These observations are consistent with the literature. As pointed out in [49], the SSFS can force solitons into an attractive state. Also, in [36] it was shown that the $\Delta \phi$ dependence of the soliton force decreases with increasing group velocity difference, eventually becoming attractive for all $\Delta \phi$.

In Figs. $\underline{4(\mathrm{a})}$ and $\underline{4(\mathrm{~b})}$ we show $\left|E_{\text {out }}(t)\right|^{2}$ and the instantaneous angular frequency when $\Delta t=500 \mathrm{fs}$ for two different $\Delta \phi$ separated by $\pi$. Here the result is independent of $\Delta \phi$, as expected, as the soliton force is negligible at this $\Delta t$. In Figs. 4(c) and $4(\mathrm{~d}), \Delta t=260 \mathrm{fs}$, and curves are given for $\Delta \phi=1.6 \pi$ and $\Delta \bar{\phi}=0.5 \pi$, corresponding to $\min \left[\Delta T_{\text {sol }}\right]$ and $\max \left[\Delta T_{\text {sol }}\right]$, respectively. These show that the two solitons,

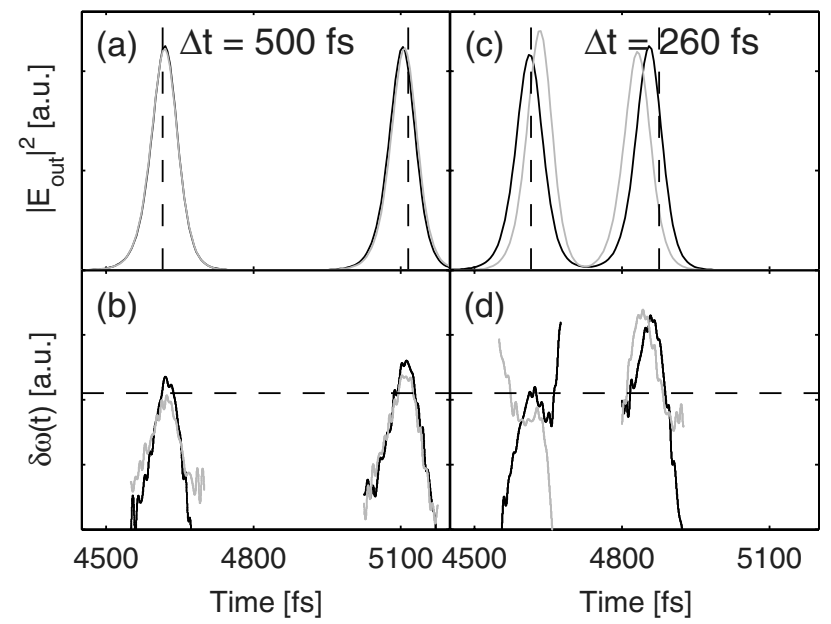

Fig. 4. Simulation; soliton pair with $\tau=44 \mathrm{fs}, \lambda=1035 \mathrm{~nm}$, and $P_{0}=1710 \mathrm{~W}$. Output temporal envelopes and instantaneous angular frequencies for (a), (b) $\Delta t=500 \mathrm{fs}$, (c), (d) $\Delta t=260 \mathrm{fs}$. $\Delta \phi=1.3 \pi$ (gray), corresponding to $\min \left[\Delta T_{\mathrm{sol}}\right] ; \Delta \phi=0.2 \pi$ (black), corresponding to $\max \left[\Delta T_{\mathrm{sol}}\right]$. Soliton positions in the noninteracting case (vertical dashed) and instantaneous angular frequency in the noninteracting case (horizontal dashed), for reference. 
though initialized as identical, do not stay identical. Rather, the leading soliton slows down and frequency downshifts relative to the trailing pulse with the frequency difference being slightly larger when $\Delta T_{\text {sol }}=\min \left[\Delta T_{\text {sol }}\right]$.

\section{B. Pair of Imperfect Solitons}

One additional effect becomes significant when $E_{0}$ is not a soliton, in which case the initial steps of propagation involve the splitting of the pulse into a soliton and a dispersive residue, the former redshifting due to the SSFS and slowing down due to dispersion and the latter staying around the initial wavelength. The leading soliton will then cross the residue of the trailing pulse. For this series of simulations, we initialize $E_{0}$ as Gaussians with duration FWHM 140 fs. A few descriptive results are presented in Figs. $5(\mathrm{a})-5(\mathrm{~d})$. We see from Figs. $5(\mathrm{~b})$ and $5(\mathrm{~d})$ that the interaction is now in general $\Delta \overline{T_{\mathrm{sol}^{-}}}$ decreasing. This is due to interaction through stimulatedRaman scattering (SRS); when the leading soliton passes through the residue, they are close enough in frequency that they can interact through the Raman response of the PCF. Because the soliton is at lower frequency, it will act as the Raman Stokes, being amplified while the residue acts as Raman pump, being depleted. This causes an increase in the leading soliton's energy, leading to a frequency downshift through the SSFS and deceleration, and hence, in effect, it is a $\Delta T_{\text {sol-decreasing in- }}$ teraction. Only when $\Delta T_{\text {sol }}$ gets close to $300 \mathrm{fs}$, which happens for $\Delta t=500 \mathrm{fs}$, Figs. $5(\mathrm{c})$ and $5(\mathrm{~d})$, the $\Delta \phi$-dependent soliton force is significant in the last part of the propagation in the PCF. We note that $\min \left[\Delta T_{\text {sol }}\right]$ occurs at $\Delta \phi=0.3 \pi$ and $\max \left[\Delta T_{\text {sol }}\right]$ at $\Delta \phi=1.3 \pi$. That $\min \left[\Delta T_{\text {sol }}\right]$ does not occur at $\Delta \phi=0$ was also
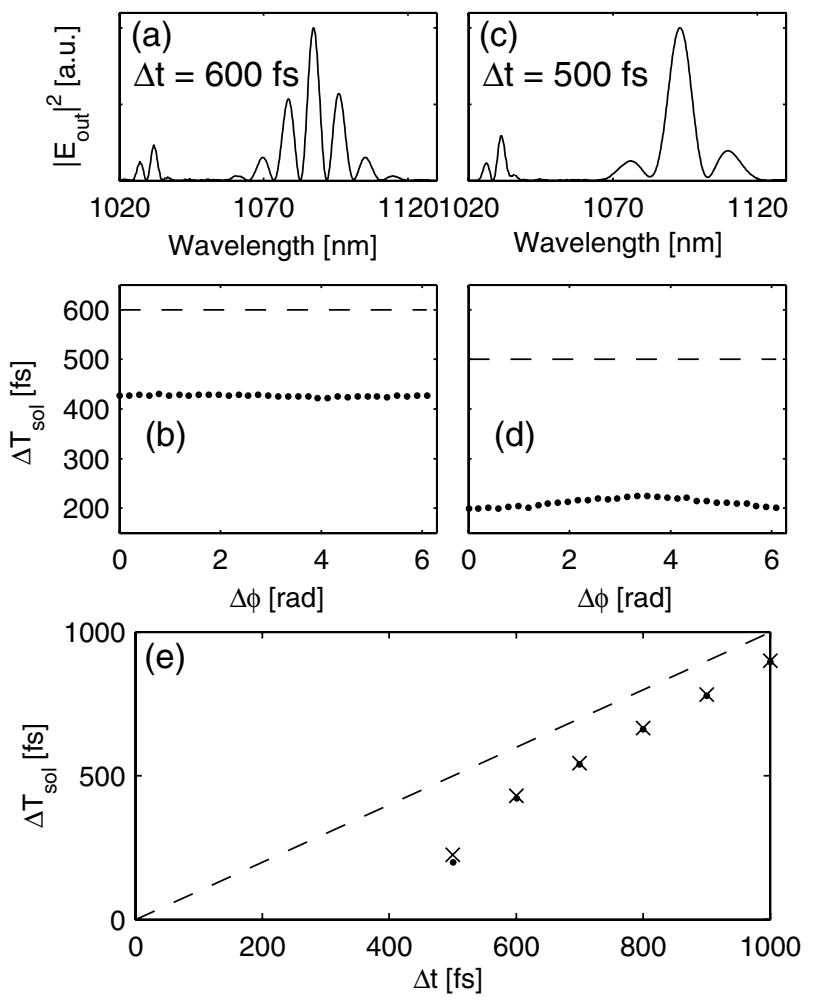

Fig. 5. Simulation; Gaussian pulse pair with $\tau=140 \mathrm{fs}$ at $\lambda=1035 \mathrm{~nm}, P_{0}=600 \mathrm{~W}$. Sample output spectra and output relative delay $\Delta T_{\text {sol }}$ versus initial relative phase $\Delta \phi$ for initial relative delay (a), (b) $\Delta t=600 \mathrm{fs},\left(\mathrm{c}\right.$ ), (d) $\Delta t=500 \mathrm{fs}$, (e) $\min \left[\Delta T_{\text {sol }}\right]$ (dots) and $\max \left[\Delta T_{\text {sol }}\right]$ (crosses) versus $\Delta t$, and the noninteracting case (dashed). seen in Subsection 3.A for perfect solitons. Here there could also be contribution from a small phase shift that happens due to the frequency shift the soliton acquires in crossing the residue. We summarize the results of several simulations for different values of $\Delta t$ in Fig. $5(\mathrm{e})$.

Figures $\underline{6(\mathrm{a})}$ and $\underline{6(\mathrm{~b})}$ show $\left|E_{\text {out }}(t)\right|^{2}$ and the instantaneous angular frequency for a $\Delta t=600 \mathrm{fs}$ where the soliton force is negligible. Nevertheless, although the two pulses were identical at the onset, when leaving the PCF, the leading pulse has acquired an increase in peak power and a frequency downshift. This is compatible with the explanation that it has gained energy from interacting with the residue of the trailing soliton. Traces for $\Delta \phi=0.3 \pi$ as well as for $\Delta \phi=1.3 \pi$ are presented in Fig. 6, demonstrating that this contribution to the integrated interaction is phase independent. The trailing soliton is unaffected, which is expected because it never crosses any other pulse. In Figs. $\underline{6(\mathrm{c})}$ and $\underline{6(\mathrm{~d})}$ are shown $\left|E_{\text {out }}(t)\right|^{2}$ and the instantaneous angular frequency for $\Delta t=500 \mathrm{fs}$, where the soliton force is significant. $\left(\max \left[\Delta T_{\mathrm{sol}}\right]-\min \left[\Delta T_{\mathrm{sol}}\right]\right)$ is less than in Subsection 3.A. Whereas in the previous case, the interaction is sufficient at $\Delta t=250 \mathrm{fs}$ to induce collision, in the present case, no collision takes place at $\Delta T_{\text {sol }}=200 \mathrm{fs}$ (which happens for $\Delta t=500 \mathrm{fs}$ ). This be seen by comparing Fig. $\underline{5(\mathrm{e})}$ with Fig. 3(e) or Fig. 6(c) with Fig. 4(c). We ascribe this to the increased difference in soliton group velocities caused by the soliton-residue crossing; as pointed out in [36], the $\Delta \phi$ dependence of the soliton force diminishes with increasing group velocity difference, eventually becoming attractive for all $\Delta \phi$.

\section{Pair of Overlapping Pulses}

If there is significant temporal overlap between the two input pulses, the interference will significantly influence the energy deposed into each formed soliton. To simulate this effect, we initialize the simulation with a pair of pulses with sech ${ }^{2}$ envelopes and FWHM $140 \mathrm{fs}$. Sech $^{2}$ pulses are characterized by approaching zero slower with $t$ than do Gaussians, which is why we chose this shape to highlight the effect of interference between the initial pulses. The FWHM and peak power were set equal to those used in the previous case, facilitating

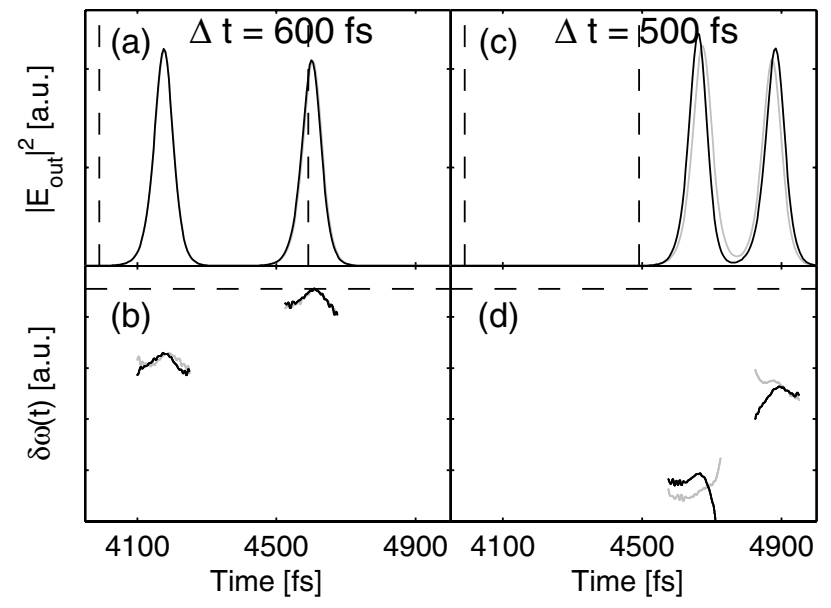

Fig. 6. Simulation; Gaussian pulse pair with $\tau=140 \mathrm{fs}$ at $\lambda=1035 \mathrm{~nm}, P_{0}=600 \mathrm{~W}$. Output temporal envelopes and instantaneous angular frequencies for (a), (b) $\Delta t=600 \mathrm{fs,} \mathrm{(c),} \mathrm{(d)} \Delta t=$ 500 fs. $\Delta \phi=0.3 \pi$ (gray), corresponding to $\min \left[\Delta T_{\text {sol }}\right] ; \Delta \phi=1.3 \pi$ (black), corresponding to $\max \left[\Delta T_{\text {sol }}\right]$. Soliton positions in the noninteracting case (vertical dashed) and instantaneous angular frequency in the noninteracting case (horizontal dashed), for reference. 

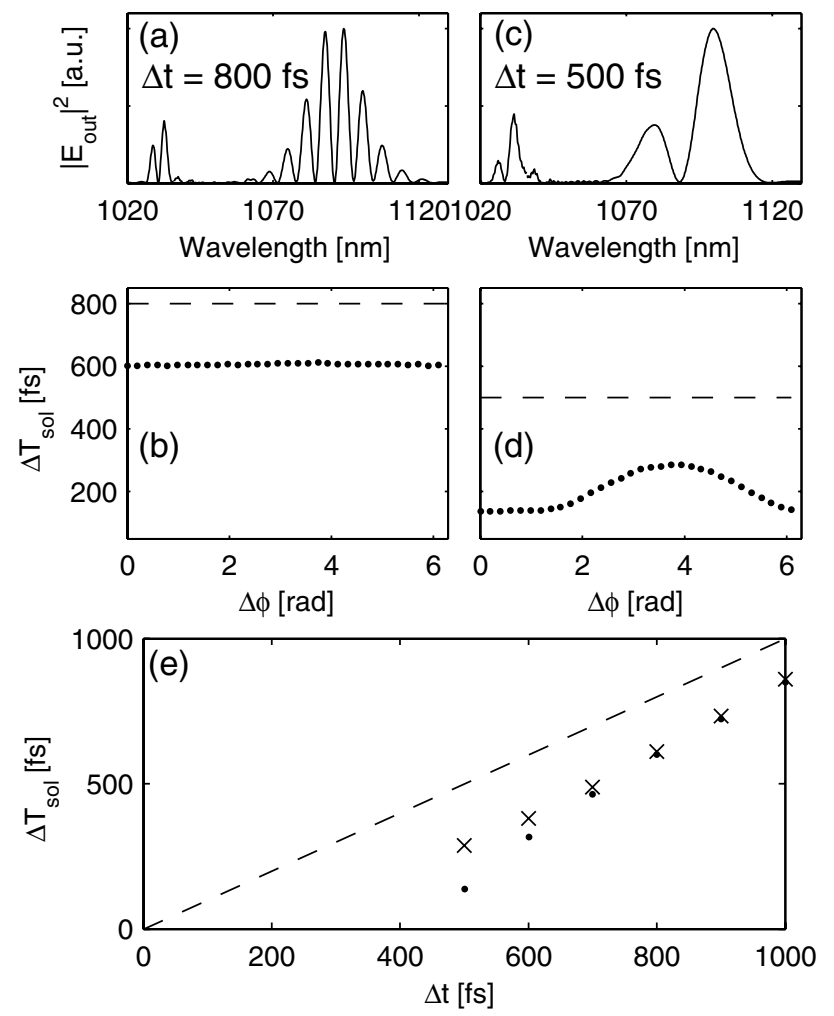

Fig. 7. Simulation; sech $^{2}$ pulse pair with $\tau=140 \mathrm{fs}$ at $\lambda=1035 \mathrm{~nm}$, $P_{0}=600 \mathrm{~W}$. Sample output spectra and output relative delay $\Delta T_{\text {sol }}$ versus initial relative phase $\Delta \phi$ for initial relative delay (a), (b) $\Delta t=800 \mathrm{fs}$, (c), (d) $\Delta t=500 \mathrm{fs,} \mathrm{(e)} \min \left[\Delta T_{\mathrm{sol}}\right]$ (dots) and $\max \left[\Delta T_{\text {sol }}\right]$ (crosses) versus $\Delta t$, and the noninteracting case (dashed). Note that, in (d), the flatness of the curve around $\Delta \phi=0$ is due to the solitons colliding, which is also why the spectrum in (c) does not look like the superposition of two soliton spectra.

comparison. Representative results in similar form as previous are given in Figs. 7(a) $-7(\mathrm{~d})$, and the outcomes of simulations for a range of $\overline{\Delta t}$ are summarized in Fig. $7(\mathrm{e})$. The conclusions to be drawn here are generally the same as for the Gaussian case; the soliton-residue crossing also here leads to

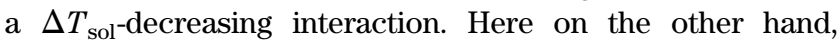
$\left(\max \left[\Delta T_{\text {sol }}\right]-\min \left[\Delta T_{\text {sol }}\right]\right)$ is larger than in Subsections 3.A and $3 . \mathrm{B}$, as seen from the increased separation of dots and crosses in Fig. 7(e) compared to Figs. $\underline{3(\mathrm{e})}$ and 5(e). Thus, there is an additional contribution to the $\overline{\Delta \phi}$-dependent interaction. This contribution stems from the interference of the constituent pulses in $E_{\text {pair }}(t)$ in interplay with the nonlinear dynamics leading up to soliton formation. The interference between the two pulses leads to two symmetrically perturbed pulse envelopes that are mirror images of each other, their shape dependent on $\Delta \phi$. Because the SSFS is not symmetric in frequency and time, the nonlinear evolutions of the two pulses are different and the perturbed pulses form solitons with different energies.

In Figs. $\underline{8(\mathrm{a})}$ and $\underline{8(\mathrm{~b})}$ are plotted $\left|E_{\text {out }}(t)\right|^{2}$ and instantaneous angular frequency for $\Delta t=800 \mathrm{fs}$, which are qualitatively similar to Figs. $\underline{6(\mathrm{a})}$ and $\underline{6(\mathrm{~b})}$. For $\Delta t=500 \mathrm{fs}$, however, Figs. $8(\mathrm{c})$ and $\underline{8(\mathrm{~d})}$, there is a difference compared to Figs. $\underline{6(\mathrm{c})}$ and $\underline{6(\mathrm{~d})}$ in that the two solitons are not equally and oppositely affected by the $\Delta \phi$ dependence. We take this as confirmation that our explanation above is correct; the soliton force alone is not responsible for the $\Delta \phi$ dependence in the case of significantly overlapping input pulses; interference in interplay with nonlinear dynamics gives a contribution to the $\Delta \phi$ dependence as well.

\section{EXPERIMENTAL RESULTS}

\section{A. $\Delta \phi$ and $\Delta t$ Dependence}

Initially, we will attempt to establish a link between the simulations and the experiments. To this end, we present the set of experiments that the simulations described above were meant to describe, launching $E_{\text {pair }}^{(\exp )}$ into the PCF, keeping $\Delta t$ fixed while scanning $\Delta \phi$. A representative PCF output spectrum is given in Fig. 9(a), the intensity autocorrelation of the laser used is shown in Fig. 9(b), and the summary of the experimental results is presented in Fig. 9(c). It is seen that the interaction is in general $\Delta T_{\text {sol-decreasing, a manifestation of the }}$ interaction through SRS between the leading soliton with the dispersive residue from the trailing pulse, in accordance with the simulations. In comparing with the simulations, the best

qualitative agreement is thus with Fig. $7(\mathrm{e})$, the most general case treated. That $\left(\max \left[\Delta T_{\mathrm{sol}}\right]-\min \left[\overline{\Delta T_{\mathrm{sol}}}\right]\right)$ stays nonzero until $\Delta t \approx 1000 \mathrm{fs}$ indicates that the temporal envelope of the laser is not completely Gaussian but has small tails. $\left(\max \left[\Delta T_{\text {sol }}\right]-\min \left[\Delta T_{\text {sol }}\right]\right)$ can be aggravated when we, as in Fig. 10, intentionally misalign the laser. The misalignment leads to the appearance of a narrow Kelly sideband in the laser spectrum and a quasi-cw component in the time domain. As such, this experiment is reminiscent of [27]. As seen in Fig. 10(b), although the FWHM is now slightly smaller, large wings of the order of some per mille in relative intensity extend way beyond the FWHM. As seen in Fig. 10(c), these small wings are sufficient to significantly increase $\left(\max \left[\Delta T_{\mathrm{sol}}\right]-\min \left[\Delta T_{\mathrm{sol}}\right]\right)$.

\section{B. Spectral Brightness Increase}

Even though many effects contribute to the interaction between pulses and solitons in the PCF, this has only a limited negative impact on the applicability of multisoliton superposi-

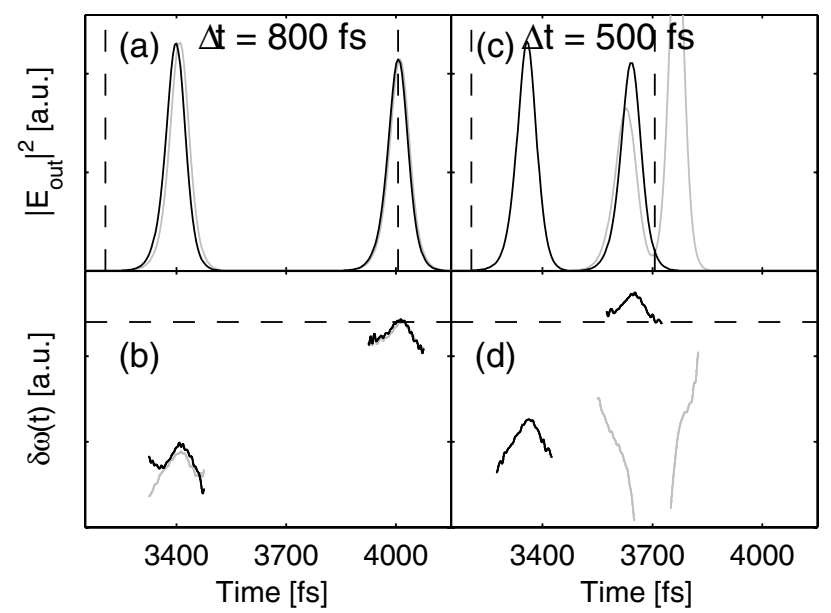

Fig. 8. Simulation; sech $^{2}$-pulse pair with $\tau=140 \mathrm{fs}$ at $\lambda=1035 \mathrm{~nm}$, $P_{0}=600 \mathrm{~W}$. Output temporal envelopes and instantaneous angular frequencies for (a), (b) $\Delta t=800 \mathrm{fs}$, (c), (d) $\Delta t=500 \mathrm{fs} . \Delta \phi=0.2 \pi$ (gray), corresponding to $\min \left[\Delta T_{\mathrm{sol}}\right] ; \Delta \phi=1.2 \pi$ (black), corresponding to $\max \left[\Delta T_{\text {sol }}\right]$. Soliton positions in the noninteracting case (vertical dashed) and instantaneous angular frequency in the noninteracting case (horizontal dashed), for reference. 

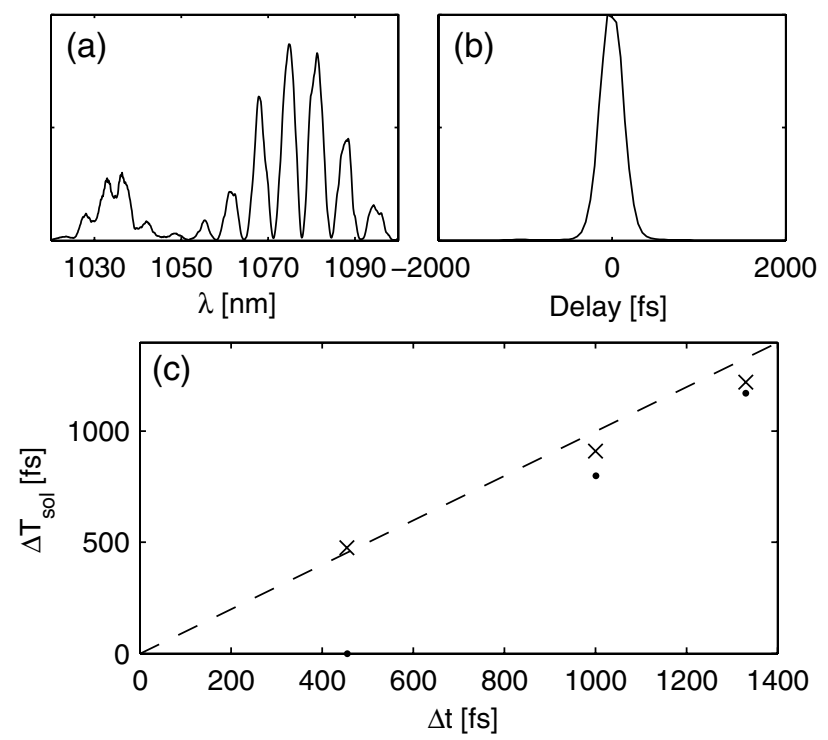

Fig. 9. Experiment; (a) representative PCF output spectrum, (b) intensity autocorrelation of the laser, (c) $\min \left[\Delta T_{\text {sol }}\right]$ (dots) and $\max \left[\Delta T_{\text {sol }}\right]$ (crosses) versus $\Delta t$, and the noninteracting case (dashed).

tion for tailoring $\left|E_{\text {out }}^{(\exp )}\right|^{2}$ in time and frequency. To this end, we now present some results of nonlinear pulse shaping, employing the aforementioned laser, for which all the discussed interactions are at play simultaneously. Figure 11(a) shows the resulting $\left|E_{\text {out }}^{(\exp )}(\omega)\right|^{2}$ when launching $E_{\text {pair }}^{(\exp )} \frac{1(\mathrm{a})}{\text { with }}$ $\Delta t=640 \mathrm{fs}$. The spectrum changes from the thick black to the thin black by changing $\Delta \phi$ by an uneven integer multiple of $\pi$. First, we emphasize that this is an example of a brightness increase of almost 4 compared to the constituent solitons (gray spectra), or a factor of 2 compared to the incoherent sum. This is due to interference between the two solitons, and in a way, the SSFS has shifted the initial interference pattern at the laser wavelength to longer wavelengths. By ex-
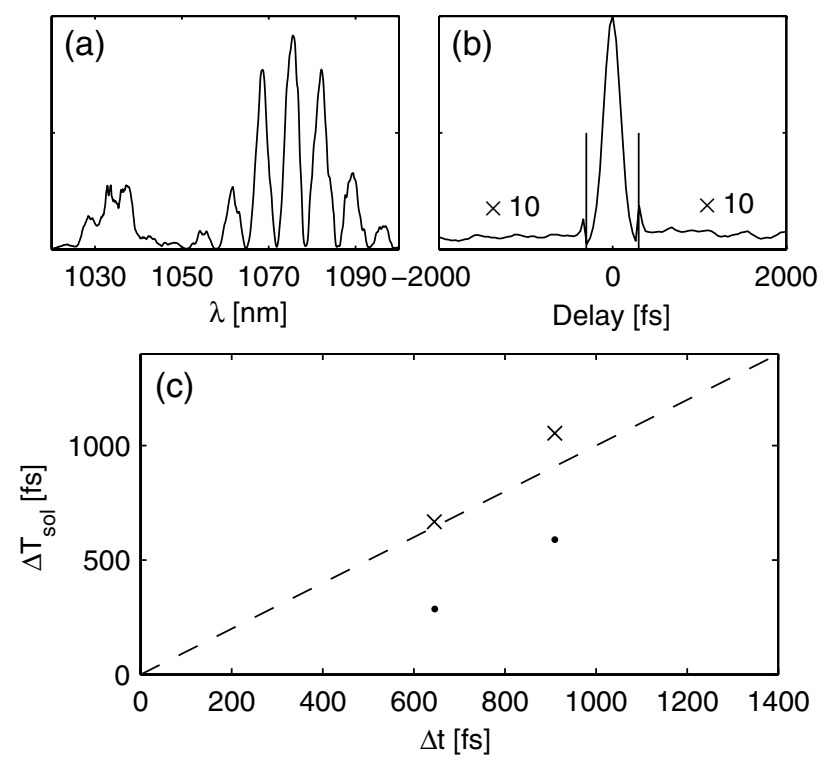

Fig. 10. Experiment with misaligned laser. (a) Representative PCF output spectrum. (b) Intensity autocorrelation of the laser; the wings are magnified to better show the quasi-cw component arising from a Kelly sideband. (c) Output relative delay $\Delta T_{\text {sol }}$ versus input relative delay $\Delta t$ for the attractive branch (dots), the repulsive branch (crosses), and the noninteracting case (dashed).
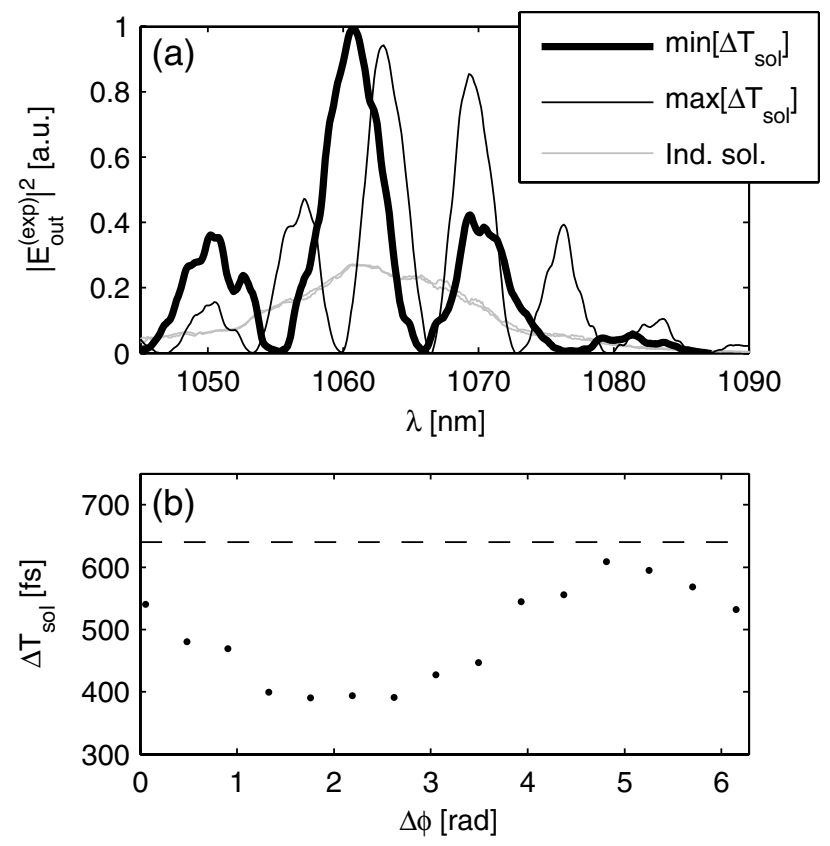

Fig. 11. Experiment; pulse pair, $\Delta t=640 \mathrm{fs}$. (a) Output spectra of the individual solitons propagating alone (gray), the soliton pair for $\min \left[\Delta T_{\text {sol }}\right]$ (thick solid), the soliton pair for $\max \left[\Delta T_{\text {sol }}\right]$ (thin solid); (b) measured $\Delta t$ (dashed) and measured $\Delta T_{\text {sol }}$ (black dots) versus $\Delta \phi$.

tension, if $n$ solitons had been launched, brightness increases compared to the fundamental soliton of $n^{2}$ would be attainable. The comparison to the brightness of a fundamental soliton is justified because its brightness is inherently limited by the fiber parameters; this limited its usefulness when used as Stokes pulse in coherent anti-Stokes Raman scattering (CARS) microspectroscopy [15]. Figure 11(b) shows the dependence of $\Delta T_{\text {sol }}$ upon $\Delta \phi$ as was expected, detailing the qualitative agreement with Figs. $5(\mathrm{~d})$ and $7(\mathrm{~d})$. In the experiment, $\min \left[\Delta T_{\text {sol }}\right]$ is found at $\Delta \phi=0.7 \pi$ due to the effects described in Section 3. Though all the effects so far discussed play a role in the interaction, the $\Delta \phi$ dependence is still benign; the soliton-residue interaction contributes a negative offset to $\Delta T_{\text {sol }}$ and decreases $\left(\max \left[\Delta T_{\mathrm{sol}}\right]-\min \left[\Delta T_{\mathrm{sol}}\right]\right)$, which is counteracted by the initial interference of the constituent pulses, which increases $\left(\max \left[\Delta T_{\mathrm{sol}}\right]-\min \left[\Delta T_{\mathrm{sol}}\right]\right)$.

\section{Phase Stability}

The solitons in the PCF add coherently, by which we mean coherent from one laser shot to the next, i.e., the phase stays unchanged from shot to shot and the output relative phase is thus a function of the input relative phase. To do this, it is sufficient to examine Fig. 11(a) and take notice of the visibility of the spectral interference fringes, which is close to $100 \%$. Now, these spectra are acquired over many millions of laser shots (seconds), so if there were any phase fluctuation from shot to shot, the visibility would be decreased. This is in line with what the simulations showed, that, in the case of correlated $P_{0}$ fluctuations, the spectral interference fringes stay where they are.

\section{CONCLUSION}

We have investigated the impact of interpulse interactions between pulse pairs $E_{\text {pair }}$ propagating in a PCF with general nonlinear response. When $E_{\text {pair }}$ is composed of solitons at the 
onset of propagation, a departure from the simplified analytical expression of [25] is observed due to the SSFS,

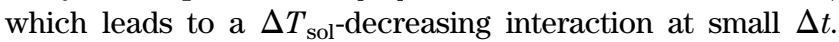
When $E_{\text {pair }}$ is not initially composed of solitons, the constituent pulses first evolve into solitons in shedding a dispersive residue; the interaction through SRS between the leading soliton and the trailing residue leads to a net decrease of $\Delta T_{\mathrm{sol}}$. If, in addition, the constituent pulses initially overlap, this gives rise to an interaction that can increase or decrease $\Delta T_{\text {sol }}$, depending on $\Delta \phi$. In our experiments, all of the mentioned effects were found to be significant. It is thus apparent from our treatment that, in order to access the most simple domain treated in Section 3.A, $E_{\text {pair }}$ must be preshaped with a phase and amplitude shaper.

As an experimental example, we demonstrated how the coherent addition of solitons in a PCF can simultaneously redshift an input pulse pair and through interference increase the spectral brightness by a factor of 2 compared to the incoherent sum. The same example demonstrated how a small difference in the relative delay at the PCF input can translate into a large change at the PCF output. Or, quoting the simulations, a change in the relative phase without any change in the temporal envelope could produce similar results, thus facilitating nonmechanical scanning of a delay over hundreds of femtoseconds.

We note the fundamental feature of the present approach to controlling the nonlinear interaction in optical fibers: in adding solitons, which are stable under propagation regardless of the magnitude of the GVD, this is a universal approach, which is not dependent on the exact fiber parameters as long as the fiber has anomalous dispersion and is sufficiently nonlinear.

Besides the obvious prospects of extending the described method to more general input pulse trains containing more than two pulses with unbalanced intensities, an interesting future potential for nonlinear pulse shaping by superposition of fundamental solitons could be to experimentally synthesize the soliton trains predicted in [37,39] to propagate as a single entity.

We believe that nonlinear pulse shaping has potential applications in nonlinear microscopy where high spectral brightness is required, such as CARS microscopy, SRS microscopy, and two-photon fluorescence microscopy.

\section{ACKNOWLEDGMENTS}

We thank Sebastien Michel for stimulating discussions. Financial support was provided by the French National Research Agency (grant SOFICARS RIB 2007), the Région ProvenceAlpes-Côte-d'Azur, and the CNRS-Weizmann NaBi European Associated Laboratory. We are grateful to Amplitude Systems for making the t-pulse laser available for the experiments.

\section{REFERENCES}

1. J. M. Dudley, G. Genry, and S. Coen, "Supercontinuum generation in photonic crystal fiber," Rev. Mod. Phys. 78, 1135-1184 (2006).

2. J. M. Dudley and J. R. Taylor, "Ten years of nonlinear optics in photonic crystal fibre," Nat. Photon. 3, 85-90 (2009).

3. A. M. Weiner, "Femtosecond pulse shaping using spatial light modulators," Rev. Sci. Instrum. 71, 1929-1960 (2000).

4. F. Verluise, V. Laude, Z. Cheng, C. Spielmann, and P. Tournois, "Amplitude and phase control of ultrashort pulses by use of an acouosto-optic programmable dispersive filter: pulse compression and shaping," Opt. Lett. 25, 575-577 (2000).
5. K. Tada and N. Karasawa, "Broadband coherent anti-Stokes Raman scattering spectroscopy using soliton pulse trains from a photonic crystal fiber," Opt. Commun. 282, 3948-3952 (2009).

6. D. Türke, W. Wohlleben, J. Teipel, M. Motzkus, B. Kibler, J. Dudley, and H. Giessen, "Chirp-controlled soliton fission in tapered optical fibers," Appl. Phys. B 83, 37-42 (2006).

7. S. Xu, D. H. Reitze, and R. S. Windeler, "Controlling nonlinear processes in microstructured fibers using shaped pulses," Opt. Express 12, 4731-4741 (2004).

8. F. Weise, M. Pawlowska, G. Achazi, and A. Lindinger, "Parametrically phase-, amplitude-, and polarization-shaped femtosecond laser pulses guided via a step-index fiber," J. Opt. Soc. Am. B 28, 406-415 (2011).

9. F. M. Mitschke and L. F. Mollenauer, "Discovery of the soliton self-frequency shift," Opt. Lett. 11, 659-661 (1986).

10. J. P. Gordon, "Theory of the soliton self-frequency shift," Opt. Lett. 11, 662-664 (1986).

11. G. P. Agrawal, Nonlinear Fiber Optics (Elsevier, 2007).

12. M. Oberthaler and R. A. Höpfel, "Special narrowing of ultrashort laser-pulses by self-phase modulation in optical fibers," Appl. Phys. Lett. 63, 1017-1019 (1993).

13. S. A. Planas, N. L. P. Mansur, C. H. B. Cruz, and H. L. Fragnito, "Spectral narrowing in the propagation of chirped pulses in single-mode fibers," Opt. Lett. 18, 699-701 (1993).

14. E. R. Andresen, J. M. Dudley, D. Oron, C. Finot, and H. Rigneault, "Transform-limited spectral compression by selfphase modulation of amplitude shaped pulses with negative chirp," Opt. Lett. 36, 707-709 (2011).

15. E. R. Andresen, V. Birkedal, J. Thøgersen, and S. R. Keiding, "Tunable light source for coherent anti-Stokes Raman scattering microspectroscopy based on the soliton self-frequency shift," Opt. Lett. 31, 1328-1330 (2006).

16. H. U. Ulriksen, J. Thøgersen, S. R. Keiding, I. R. Perch-Nielsen, J. S. Dam, D. Z. Palima, H. Stapelfeldt, and J. Glückstad, "Independent trapping, manipulation and characterization by an all-optical biophotonics workstation," J. Eur. Opt. Soc. 3, 08034 (2008).

17. V. P. Mitrokhin, A. B. Fedotov, A. A. Ivanov, M. V. Alfimov, and A. M. Zheltikov, "Coherent anti-Stokes Raman scattering microspectroscopy of silicon components with a photonic-crystal fiber frequency shifter," Opt. Lett. 32, 3471-3473 (2007).

18. G. McConnell and E. Riis, "Photonic crystal fibre enables shortwavelength two-photon laser scanning fluorescence microscopy with fura-2," Phys. Med. Biol. 49, 4757-4763 (2004).

19. A. B. Fedotov, A. A. Voronin, A. A. Ivanov, and A. M. Zheltikov, "Spectral compression of frequency-shifting solitons in a photonic-crystal fiber," Opt. Lett. 34, 662-664 (2009).

20. A. Apolonski, B. Povazay, A. Unterhuber, W. Drexler, W. J. Wadsworth, J. C. Knight, and P. S. J. Russell, "Spectral shaping of supercontinuum in a cobweb photonic-crystal fiber with sub20 fs pulses," J. Opt. Soc. Am. B 19, 2165-2170 (2002).

21. Z. Zhu and T. G. Brown, "Effect of frequency chirping on supercontinuum generation in photonic crystal fibers," Opt. Express 12, 689-694 (2004).

22. D. R. Austin, J. A. Bolger, C. M. de Sterke, and B. J. Eggleton, "Narrowband supercontinuum control using phase shaping," Opt. Express 14, 13142-13150 (2006).

23. A. A. Voronin, I. V. Fedotov, A. B. Fedotov, and A. M. Zheltikov "Spectral interference of frequency-shifted solitons in a photonic-crystal fiber," Opt. Lett. 34, 569-571 (2009).

24. J. P. Gordon, "Interaction forces among solitons in optical fibers," Opt. Lett. 8, 596-598 (1983).

25. F. M. Mitschke and L. F. Mollenauer, "Experimental observation of interaction forces between solitons in optical fibers," Opt. Lett. 12, 355-357 (1987).

26. Y. Kodama and K. Nozaki, "Soliton interaction in optical fibers," Opt. Lett. 12, 1038-1040 (1987).

27. W. H. Loh, A. B. Grudinin, V. V. Afanasjev, and D. N. Payne, "Soliton interaction in the presence of a weak nonsoliton component," Opt. Lett. 19, 698-700 (1994).

28. N. Akhmediev, W. Krolikowski, and A. J. Lowery, "Influence of the Raman-effect on solitons in optical fibers," Opt. Commun. 131, 260-266 (1996).

29. M. Romagnoli, L. Socci, M. Midrio, P. Franco, and T. Georges, "Long-range soliton interactions in dispersion-managed links," Opt. Lett. 23, 1182-1184 (1998). 
30. K. Smith and L. F. Mollenauer, "Experimental observation of soliton interaction over long fiber paths: discovery of a long-range interaction," Opt. Lett. 14, 1284-1286 (1989).

31. A. Efimov, A. V. Yulin, D. V. Skryabin, J. C. Knight, N. Joly, F. G. Omenetoo, A. J. Taylor, and P. S. J. Russell, "Interaction of an optical soliton with a dispersive wave," Phys. Rev. Lett. 95, 213902 (2005).

32. C. Rotschild, B. Alfassi, O. Cohen, and M. Segev, "Long-range interactions between optical solitons," Nat. Phys. 2, 769-744(2006).

33. F. Luan, D. V. Skryabin, A. V. Yulin, and J. C. Knight, "Energy exchange between colliding solitons in photonic crystal fibers," Opt. Express 14, 9844-9853 (2006).

34. A. Podlipensky, P. Szarniak, N. Y. Joly, C. G. Poulton, and P. S. J. Russell, "Bound soliton pairs in photonic crystal fiber," Opt. Express 15, 1653-1662 (2007).

35. A. Hause, H. Hartwig, M. Böhm, and F. M. Mitschke, "Binding mechanism of temporal soliton molecules," Phys. Rev. A 78, 063817 (2008)

36. A. Hause and F. M. Mitschke, "Reduced soliton interaction by Raman self-frequency shift," Phys. Rev. A 80, 063824 (2009).

37. A. Hause and F. M. Mitschke, "Soliton trains in motion," Phys. Rev. A 82, 043838 (2010).

38. A. Hause, T. X. Tran, F. Biancalana, A. Podlipensky, P. S. J. Russell, and F. M. Mitschke, "Understanding Raman-shifting multipleak states in photonic crystal fibers: two convergent approaches," Opt. Lett. 35, 2167-2169 (2010).

39. T. X. Tran, A. Podlipensky, P. S. J. Russell, and F. Biancalana, "Theory of Raman multipeak states in solid-core photonic crystal fibers," J. Opt. Soc. Am. B 27, 1785-1791 (2010).
40. T. I. Lakoba and D. J. Kaup, "Influence of the Raman effect on dispersion-managed solitons and their interchannel collisions," Opt. Lett. 24, 808-810 (1999).

41. M. Erkintalo, G. Genty, and J. M. Dudley, "On the statistical interpretation of optical rogue waves," Eur. J. Phys. Spec. Top. 185, 135-144 (2010).

42. Q. M. Nguyen and A. Peleg, "Resolving the Raman-induced cross frequency shift in fast optical soliton collisions,” J. Opt. Soc. Am. B 27, 1985-1990 (2010).

43. B. A. Malomed, "Soliton-collision problem in the nonlinear Schrödinger equation with a nonlinear damping term," Phys. Rev. A 44, 1412-1414 (1991).

44. M. Erkintalo, G. Genty, and J. M. Dudley, "Experimental signatures of dispersive waves emitted during soliton collisions," Opt. Express 18, 13379-13384 (2010).

45. M. H. Frosz, O. Bang, and A. Bjarklev, "Soliton collision and Raman gain regimes in continous-wave pumped supercontinuum generation," Opt. Express 14, 9391-9407 (2006).

46. M. Erkintalo, G. Genty, and J. M. Dudley, "Giant dispersive wave generation through soliton collision," Opt. Lett. 35, 658660 (2010).

47. S. Chi and S. Wen, "Raman cross talk of soliton collision in a lossless fiber," Opt. Lett. 14, 1216-1218 (1989).

48. S. R. Friberg, "Soliton fusion and steering by the simultaneous launch of two different color solitons," Opt. Lett. 16, 14841486 (1991).

49. E. L. Buckland, R. W. Boyd, and A. F. Evans, "Observation of a Raman-induced interpulse phase migration in the propagation of an ultrahigh-bit-rate coherent soliton pulse train," Opt. Lett. 22, 454-456 (1997). 